\title{
CONSIDERATION OF HUMAN COMPUTER INTERACTION IN ROBOTIC FIELD
}

\author{
Tarek Toumi and Abdelmadjid Zidani \\ Computer Science Department, University of Batna, 05000, Algeria
}

\begin{abstract}
Technological progress leads the apparition of robot in human environment; we began to find them in hospitals, museums, and homes. However these situations require an interaction of robots with humans and an adoption of social behaviors. We have shown in this paper how disciplines like computer science in general and human computer interaction in particular are used to improve human robot interaction. Then we indicated how we can use action theory into design of interaction between human and Robot. Finally we proposed some practical scenarios for illustrations.
\end{abstract}

\section{KEY WORDS}

Human Computer Interaction, Human Robot Interaction, Social Interaction.

\section{INTRODUCTION}

The study of human-robot interaction is a difficult task, Even the technological advances and research efforts used in this area, it would be still helpful to inspire ideas from Human-Human interaction in collaborative tasks and from Human-machine interaction to improve the interaction between robot and human.

The classical methods of human-robot interaction were fundamentally based on simple models where human controls robot by different commands, to this approach succeeds today a new vision considering robot as companion or assistant rather than an object that we can control. We begin to find robots in hospitals [1], homes [2], classrooms [3], museums [4], etc., however to be accepted in human environment, robotic systems must respond to this new vision, both by adapting robots to the increasing needs of humans and by taking into account a highly evolution technology. Unfortunately, current methods and software tools for human-robot interaction fail to satisfice these requirements easily.

An interaction model is a set of basic principles that show how the user interacts with his machine [5]. Empirical experiments based on observations and experiences have provided rich studies about relationships and collaboration between individuals in team, however due to the lack of appropriate theories, the expertise in this field cannot be generalized. It is therefore difficult to apply to new situations. Currently, there is no ready solution to use, and there are only some compromises to practice by common sense.

Action theory proposed by D. Norman [6] is used to model various cognitive steps used in performing a task. This paper, explore the cognitive steps of action theory used in the interaction process. The concept of emotion and the capabilities of the robot are introduced in the interaction 
process to improve the interaction between human and robot. Finally some human-robot interaction scenarios are used to clarify this approach.

\section{HUMAN COMPUTER INTERACTION MODELS}

Computer science has a key role in advancement of robotics in general and in human robot interaction in particular. Computer science represents the intelligence part of robot, and can transform whatever was possible to analyze it to a program that can by its turn improve the behavior of robot. In reality it is easy to see the contributions of computer science in the interaction between robot and human. That starts from the vision systems going to the social behavior of robot.

Human Computer interaction models describe how human interacts with machines, several models are proposed in the literatures, and by the following we cited the principal ones:

- Action theory proposed by D. Norman [6] is used to model various cognitive steps used in performing a task. This approach will be detailed later.

- Human Processor Model proposed by Card: this model [7] is a simplified model of human interaction process with computer systems. This model is based on three interrelated subsystems: sensory system, motor system and cognitive system. Other analysis methods of human behavior as GOMS and its simplified version Keystroke are extensions of this model.

- Interacting Cognitive Subsystems model (ICS) proposed by Bernard [8] is an extension of Human Processor Model [7]. The Interacting Cognitive Subsystems model consists of nine specialized sub-systems with a parallel-multithreaded architecture; their mechanisms of information processing are organized in modular structure.

- The instrumental theory of Rabardel [9] refers to the relationship between a user, instrument and the object to which the action was taken, i.e. it is based on the concept of the instrument of interaction, intermediary between the user and the objects it manipulates [5]. This theory of instrumental interaction is the basis for other interaction models such as that of Fuchs [10] used in virtual reality.

- Interactional process proposed by Rasmussen [11]: This model completes the action theory of Norman, by integrating several approaches to offer paradigms in risk analysis, including the SRK model (Skill, Rules, Knowledge). It offers three levels of control of human behavior: The behavior based on knowledge (knowledge), the behavior based on rules (processes) and the behavior-based skills (reflexes)

- Physical interaction Model of Sato [12]: The purpose of this model is to mix the elements of the real world "physical space", and the elements of the digital world "virtual world" to model interactive systems. It is based on a user-centered approach to produce systems that satisfice the needs of users in cognitive, cultural and social plans, and this is due to the complexity of integrating the two areas: real and virtual spaces.

- The architectural model of Seeheim [13] separates between the interface and the functional core, its interface is composed of three units: Functional Core that includes all features of the domain. Presentation: to make visible the status of domain concepts and manipulation by the user, and the Dialog Controller that serves as a bridge between the Functional Core and the Presentation, it is responsible for managing the relationship between these two units. 
- The Arch model [14] is an extension of the Seeheim model previously cited. It consists of five components organized in the form of an arch: the Functional Core, the Adapter Domain, the Dialog Controller, Component Presentation and the component of interaction.

- Multi-agents Models: many models of human machine interaction have been proposed under the multi-agent approach to provide more efficient organization. One example is the MVC (Model-View-Controller) model. PAC (presentation-Abstraction- Control) proposed for the first time by J. Coutaz [15] is also based on multi-agent approach.

\section{CONCEPTUAl Model: ACTION TheORY}

A conceptual model is a mental representation of knowledge in terms of psychological variables. Psychological variable is related to a concept, a unit of knowledge, or a topic of interest. For example, in the task of exchange an object between human and robot, the concepts of distance, height, weight and object size, etc., are specific psychological variables related to the action of object exchange.

The action theory [6] introduced the notion of conceptual model of different cognitive steps involved in an action.

- The first step in performing an action is to establish a goal, for example, the transfer of an object to the human. In this interaction between Human and Robot, the goal is a mental representation of desired state of the robot (by handing the object comfortably and in the right place, the right height, etc.). This state is represented by physical variables, i.e. the information produced by the robot through its mobility and gestures. Thus, in human-robot interaction field, the position of robot arm and the position of human hand in 3D space are physical variables that are managed by robot and are perceptible by human.

- The second step is the formulation of an intention. It expresses the basic actions to be undertaken to achieve the goal. It is therefore the distance between the goal and mental representation. This representation is the product of the translation of the state of each of two actors in psychological terms and the comparison of this result with the intended goal. Noting that the translation is possible only if the conceptual model includes knowledge of the correspondence between physical and psychological variables. For example, the robot must combine physical variable "human hand position" to the concept of targeted contact point.

- The third step is the construction of an action plan. Its execution is expected to achieve the goal. The implementation of this action plan requires the knowledge of the relationship between psychological variables involved in the goal and physical variables.

- The fourth step is the execution of the action plan by robot.

- The mutual perception of modified state and the interpretation of modified physical variables in psychological terms, results in a mental representation of new state of the system is compared to the goal. This evaluation may result in the continuation of the plan or modification.

These steps inspired from the action theory identify the key points. In particular, they highlight the existence of the translation process where the success depends on the mapping between psychological variables and physical variables as well as the establishment of associations between physical variables and robot control devices. The consequence of this observation is the design of a human-robot interaction process that promotes the establishment of such relations. 


\section{INTERACTION WITH ASSISTANTS ROBOTS: ADAPTATION OF ACTION THEORY}

In terms of theoretical contributions, a theory explains a phenomenon or predicts the occurrence. In cognitive ergonomics field, there is no single explanatory or predictive theory that can cover all aspects of human subject.

There are a multitude of particular theories; each one studies a class of specific phenomena such as problem solving theories, mental representation theories, learning theory, and many others.

Each theory provides a particular class of concepts and cognitive processes; however the dispersion leads to an impressive amount of partial studies. This is probably tolerable for cognitive researcher who is interested in a specific aspect of behavior. It is not for the computer scientist who must consider the needs of a human without dividing into functional parts!

Despite the lack of the cohesion and despite the limited application field, theoretical models such as human processor model and action theory define a useful framework.

By changing the model of D. Norman after using the feelings and abilities of the robot, the new model (Figure.1) get to be as taking after:

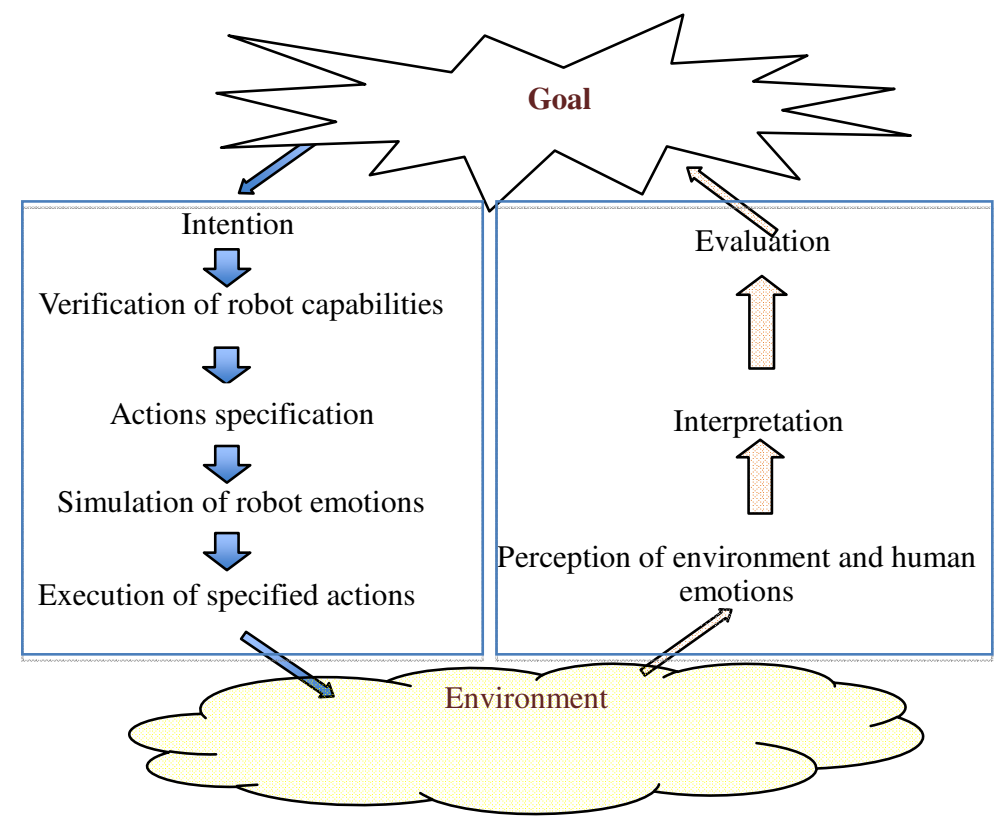

Figure 1. Norman model adapted

\section{SCENARIOS OF ADAPTED MODEL}

By following we gave a few applications situations:

This introductory situation showed social comportment of robot: 


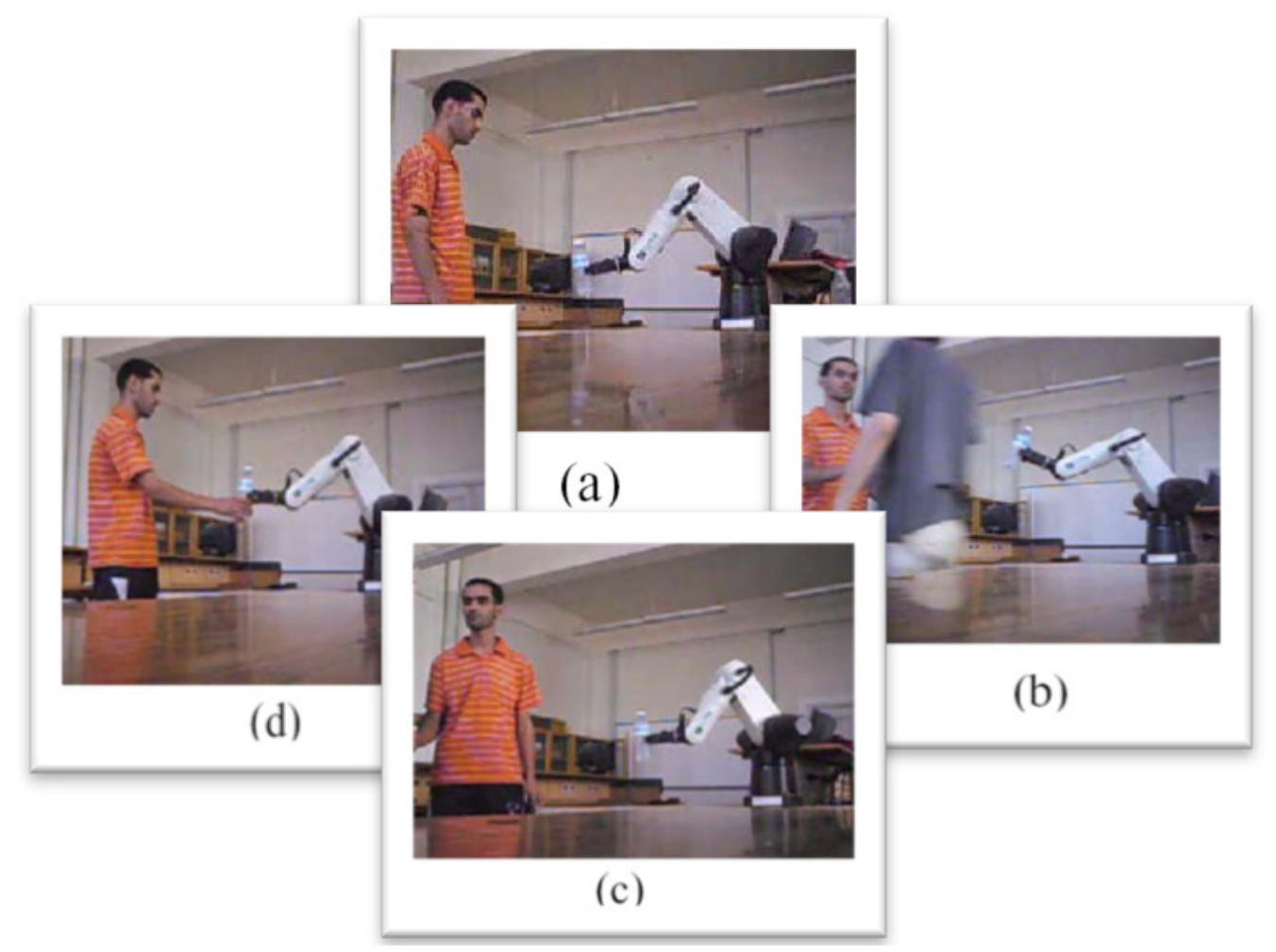

Figure 2. Social comportment of robot: (a) robot gives an object to human, (b) human is perturbed, (c) robot stops and suspends the mission and drop the object, (d) robot will perform the mission when human will be free.

The adjusted model used for this application is:

- Objective or goal: Give an object to human.

- Intention: help human.

- Verification of robot abilities: robot has all abilities to achieve the task.

- Action plan: detection of human position, path planning and trajectory generation,

- Simulation of robot feelings: supposing that the robot is in good feeling and doing his action legitimately.

- Execution: the robot achieves the action plan.

- Awareness of the environment and human feelings: robot perceives that human shakes hands with his companion.

- Interpretation: the robot interprets that human is disturbed.

- Evaluation: the robot assesses that the mission is incidentally suspended, and it ought to bring down the object. This makes another arrangement of goals when human will be free.

In this second situation we showed the cooperation of robot: 
International Journal of Computer Science \& Information Technology (IJCSIT) Vol 7, No 4, August 2015

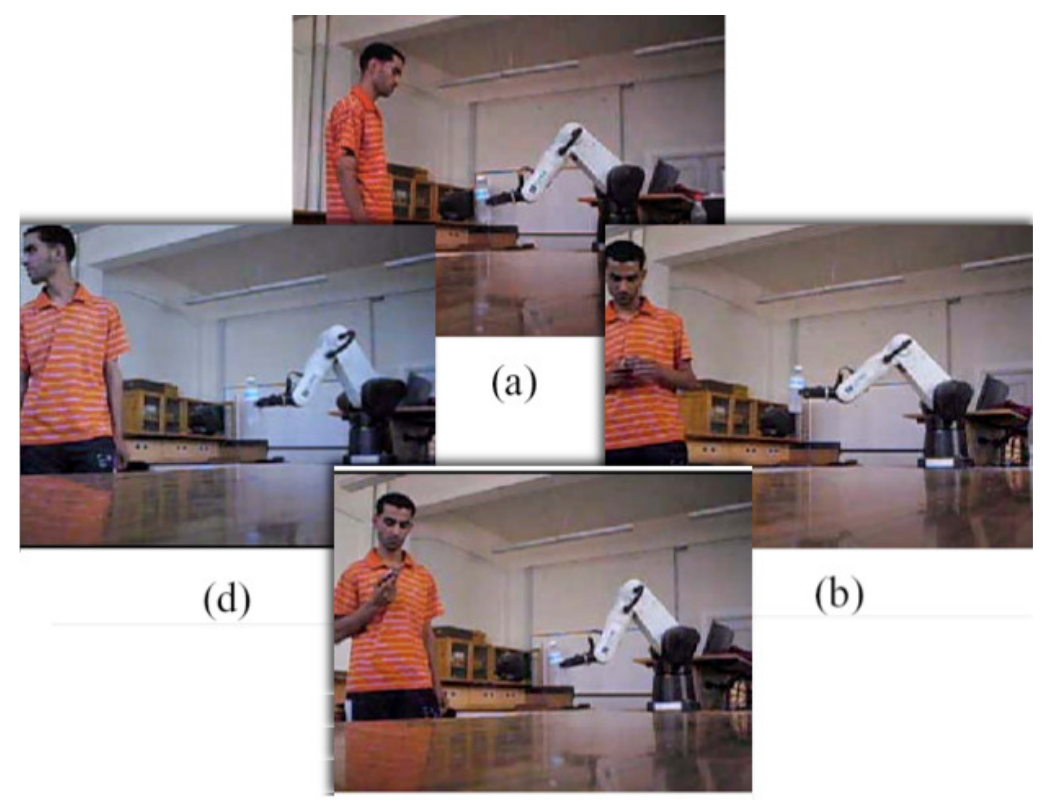

(c)

Figure 3. The human do not take the object: (a) robot gives the object to human, (b) human do not take the object, (c) robot drops the object, (d) robot leaves the mission.

Taking this third scenario (Fig.4) in which a teacher is trying to write in a whiteboard, he suddenly realizes that he made a mistake, then he wants to clean the whiteboard, in this case the robot plays the role of an assistant and give him the brush.

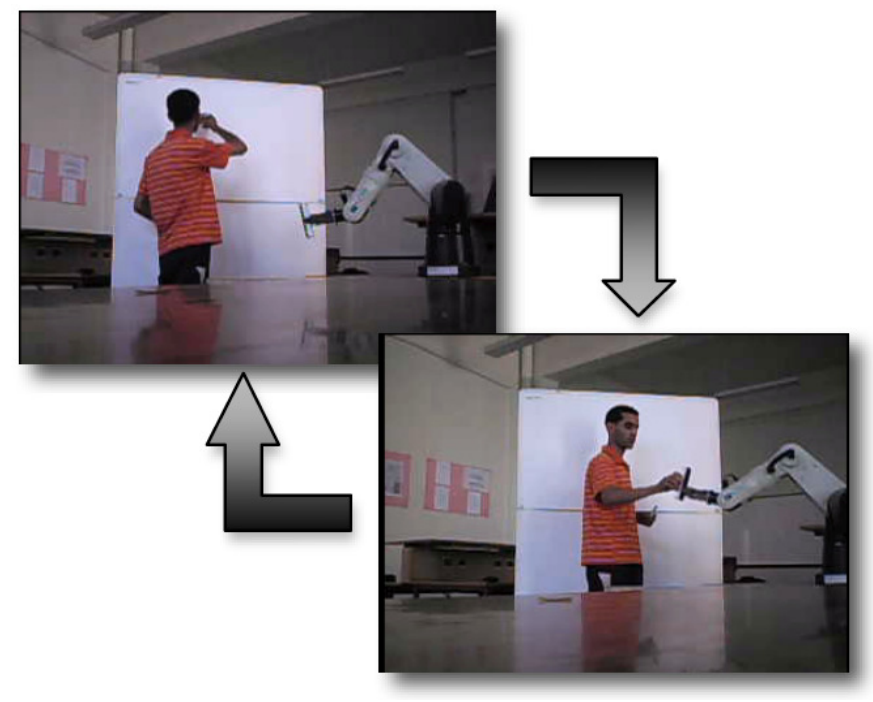

Figure 4. Scenario of Human robot interaction

The design task can be divided into three main subtasks: human identification, definition of tasks and specific objects and experimental evaluation. This diagram is a simplified view of the design process. In practice, the subtasks are: 
- Human identification: detection of human position.

- Definition of specific tasks and objects: the task of writing and brush like object.

- Awareness (Awareness of what is happening: Evaluation of human state, if he wants to brush or no?

The Awareness concept typically from fundamental factors relating to the Human robot interaction, that each party must be aware of any information that could be exploited to achieve the final goal. An example of such information that is vital to the process of interaction between human and robot:

- What is going on? (Interpretation of the current state like positions, actions, etc.).

- Where is my partner? (Location in space)

- What happens and why? (Mutual Interpretation of running actions, collaboration requires each partner to avoid being surprised by the actions of his partner, etc.

It is particularly based on a mutual perception of the attitude of each of both sides human and robot. In the first sense, human has a gigantic potential of perception and interpretation that we cannot completely simulate in the side of the robot.

\section{Confronts}

To create robots that cooperate socially with us, we have first to see how we do it as humans, regardless of the possibility that we can interact naturally; it is still difficult for robot to do it.

The following represents primary parts of the past model: testing the capacity of the robot, mimicking of robot feelings and awareness of human feelings, and attempting to make an extension between the ways that human do it and how the robot ought to mimic it.

- Testing the capacity of the robot: We can anticipate our ability in the vast majority of circumstances. Case in point, somebody can say that he can't drive an auto, even before attempting to drive it, because he knows his capacity. As indicated by the investigations of Dr. Daryl J. Bem, we can anticipate future things [16]. Concerning robots, he retains all his past objectives and results (effectively or not) to accomplish them in a database. This could be put away in the database in which all necessaries components of robot goals are recorded.

- The social perspective is a vital normal for individual. We have a gigantic ability to express our emotions through facial and physical expressions, and we have capacities to comprehend these expressions. Facial appearances express our sentiments could be utilized as a part of the statement of human emotions. There are numerous robots that can produce emotional expressions.

- The interpretation of facial and physical expressions of others has a close relationship with memory, knowing the personality of a person helps to read his precise physical and facial expressions by linking them with the previous expressions in memory.

- The understanding of facial and body language of others has an association with memory and the past facial and body language, by connecting them with the past expressions in memory.

- The robot must understand human emotions to be socially accepted by him. Chastagnol et al [17] are in light to create a module for distinguishing feeling in voice control framework for a social robot. 
International Journal of Computer Science \& Information Technology (IJCSIT) Vol 7, No 4, August 2015

\section{CONCLUSION}

Human robot interaction is an interesting field of research due the evolution of robots technologies. Meany researches focused on the best ways to use robots as companions or assistants to humans. This paper, explains the use of human computer interaction to improve human robot interaction and how we can integrate emotions and capabilities into robot design process basing on specific situations of interaction between human and Robot. Challenges related to the proposed method are briefly discussed with an illustration through real cases.

\section{REFERENCES}

[1] K. I. Kang, S. Freedman, M. J. Mataric, M. J. Cunningham, and B. Lopez, "A hands-off physical therapy assistance robot for cardiac patients," in Rehabilitation Robotics, 2005. ICORR 2005. 9th International Conference on, 2005, pp. 337-340.

[2] M. Beetz, F. Stulp, B. Radig, J. Bandouch, N. Blodow, M. Dolha, A. Fedrizzi, D. Jain, U. Klank, and I. Kresse, "The assistive kitchen-a demonstration scenario for cognitive technical systems," in Robot and Human Interactive Communication, 2008. RO-MAN 2008. The 17th IEEE International Symposium on, 2008, pp. 1-8.

[3] H. Admoni and B. Scassellati, "Roles of Robots in Socially Assistive Applications."

[4] W. Burgard, A. B. Cremers, D. Fox, D. Hähnel, G. Lakemeyer, D. Schulz, W. Steiner, and S. Thrun, "Experiences with an interactive museum tour-guide robot," Artificial intelligence, vol. 114, pp. 3-55, 1999.

[5] M. Beaudouin-Lafon, "Instrumental interaction: an interaction model for designing post-WIMP user interfaces," in Proceedings of the SIGCHI conference on Human factors in computing systems, 2000, pp. 446-453.

[6] D. A. Norman and S. W. Draper, User Centered System Design; New Perspectives on Human-Computer Interaction: L. Erlbaum Associates Inc., 1986.

[7] S. K. Card, T. P. Moran, and A. Newell, "The Psychology of Human Computer Interaction Hillsdale," NJ: LEA, 1983.

[8] P. Barnard, "Cognitive Resources and the Learning of Computer Dialogs, Interfacing Thought, Cognitive aspects of Human Computer Interaction," Carroll J. Ed., MIT Press, pp. p.112-158, 1987.

[9] P. Rabardel, Les hommes et les technologies: approche cognitive des instruments contemporains: Armand Colin Paris, 1995.

[10] J. M. Fuchs and G. et Papin, "JP (2001), Le traité de la réalité virtuelle," Les Presses de 1'Ecole des Mines, Nantes.

[11] J. Rasmussen, Information processing and human-machine interaction. An approach to cognitive engineering: North-Holland, 1987.

[12] K. Sato and Y.-k. Lim, "Physical interaction and multi-aspect representation for information intensive environments," in Robot and Human Interactive Communication, 2000. RO-MAN 2000. Proceedings. 9th IEEE International Workshop on, 2000, pp. 436-443.

[13] M. Green, "Report on dialogue specification tools," in Computer Graphics Forum, 1984, pp. 305-313.

[14] L. Bass, R. Faneuf, R. Little, N. Mayer, B. Pellegrino, S. Reed, R. Seacord, S. Sheppard, and M. R. Szczur, "A metamodel for the runtime architecture of an interactive system," SIGCHI Bulletin, vol. 24, pp. 32-37, 1992.

[15] J. Coutaz, "PAC, an implementation model for dialog design," in Proceedings of INTERACT, 1987, pp. 431-436.

[16] D. J. Bem, "Feeling the future: experimental evidence for anomalous retroactive influences on cognition and affect," Journal of personality and social psychology, vol. 100, p. 407, 2011.

[17] C. Chastagnol, C. Clavel, M. Courgeon, and L. Devillers, "Designing an emotion detection system for a socially intelligent human-robot interaction," in Natural Interaction with Robots, Knowbots and Smartphones, ed: Springer, 2014, pp. 199-211. 